\title{
Perioperative fluid dynamics evaluated by bioelectrical impedance analysis predict infectious surgical complications after esophagectomy
}

Shuichiro Oya ${ }^{1 *}$ D, Hiroharu Yamashita ${ }^{1}$, Ryohei Iwata ${ }^{1}$, Koichiro Kawasaki ${ }^{1}$, Asami Tanabe ${ }^{1}$, Koichi Yagi $^{1}$, Susumu Aikou ${ }^{2}$ and Yasuyuki Seto ${ }^{1}$

\begin{abstract}
Background: Transthoracic esophagectomy, among the most invasive surgeries, is highly associated with postoperative infectious complications which adversely affect postoperative management including fluid dynamics. The aim of the study is to evaluate the utility of perioperative bioelectrical impedance analysis (BIA) measurements for the patients after transthoracic esophagectomy.

Method: Multi-frequency BIA measurements were conducted in 24 patients undergoing transthoracic esophagectomy preoperatively, at $1 \mathrm{~h}$ after surgery, and twice daily for the following 7 days. The amounts of extracellular water (ECW), internal cellular water (ICW), total body water (TBW), and fat-free mass (FFM) were calculated. Changing trends in variables were analyzed, and the patients were subdivided according to the presence of infectious surgical adverse events to identify differences in fluid dynamics.

Results: ECW was the major body fluid compartment showing an increase after surgery, and peaked on postoperative day (POD) 2. Twelve patients experienced infectious complications. The peaks of changes in ECW and ECW/TBW appeared earlier and their values at the highest peak were significantly lower in the group without infectious complications on POD 2. The ICW/FFM value showed a mild decrease as compared to POD1 and then gradually recovered. It was significantly lower even before surgery and showed the most significant stratification on POD2. ECW/TBW of $48 \%$ and ICW/FFM of $37 \%$ on POD2 were predictive cut-off values for infectious adverse events with high area-under receiver operating characteristic (ROC) curves: 0.80 or higher.
\end{abstract}

Conclusion: BIA measurements are useful for monitoring fluid retention and may predict infectious complications in the early phase after transthoracic esophagectomy.

Trial registration: Registry name: UMIN-CTR, ID: UMIN000030734, Registered on January 9, 2018, retrospectively registered.

Keywords: Esophagectomy, Body fluid balance, Bioimpedance analysis, Postoperative complications, Infectious complications

\footnotetext{
* Correspondence: shiooya-tky@umin.ac.jp

${ }^{1}$ Department of Gastrointestinal Surgery, The University of Tokyo, 7-3-1,

Hongo, Bunkyo-ku, Tokyo 113-8655, Japan

Full list of author information is available at the end of the article
}

(c) The Author(s). 2019 Open Access This article is distributed under the terms of the Creative Commons Attribution 4.0 International License (http://creativecommons.org/licenses/by/4.0/), which permits unrestricted use, distribution, and reproduction in any medium, provided you give appropriate credit to the original author(s) and the source, provide a link to the Creative Commons license, and indicate if changes were made. The Creative Commons Public Domain Dedication waiver (http://creativecommons.org/publicdomain/zero/1.0/) applies to the data made available in this article, unless otherwise stated. 


\section{Background}

Understanding the fluid dynamics modulated by surgical stress is essential to managing perioperative patients. Body fluids begin to shift, after a surgical intervention, from the intravascular to the interstitial compartment. Thereby, these fluids do not contribute to blood circulation due to destruction of the endothelial glycocalyx, a key vascular barrier structure [1]. As the patient recovers from surgical stress, the body fluids in the interstitial compartment begin to redistribute into the intravascular compartment, i.e. fluid retention manifests. This postoperative event generally occurs within a few days and the extent of fluid dynamic changes reflects the invasiveness of the surgical procedure. Therefore, it is mandatory, especially in patients who have undergone invasive surgery, to carefully manage the infusion volume especially during the fluid redistribution phase. Preventing fluid overload as well as the resultant burden on the respiratory and cardiovascular systems is essential to avoiding a variety of postoperative adverse events.

Patients with systemic inflammatory responses secondary to infectious complications usually require more fluid administration to maintain adequate circulation during the postoperative period. The circulation is partially supported by immediate fluid resuscitation, which should reportedly be carried out within the first three hours to prevent sepsis-induced hypo-perfusion [2]. Therefore, the extent of fluid administration and subsequent retention is potentially greater in post-surgery patients with infectious complications, even before they manifest clinically, potentially necessitating even more meticulous management thereafter.

Transthoracic esophagectomy is one of the most invasive surgeries and is associated with high rates of morbidities including pneumonia, anastomotic leakage, and surgical site infection [3]. Given that this procedure is associated with not only high invasiveness but also a high incidence of infectious complications, postoperative fluid management is often a challenging issue in this population. Consequently, fluid restriction, which is more likely to achieve good outcomes than less strict fluid management strategies $[4,5]$, is not widely employed.

As a novel tool for evaluating body fluids, BIA measurements have been attracting considerable attention in the fields of nutritional assessment $[6,7]$ and critical care $[8$, 9]. BIA measurements can be employed for perioperative fluid management as well, and the usefulness of this approach has been reported [10-15]. This study aimed to assess the trends in perioperative fluid distribution changes, by employing multi-frequency BIA measurements. We also evaluated possible differences according to the presence of infectious adverse events in patients who had undergone transthoracic esophagectomy.

\section{Patients and methods}

\section{Patients}

Twenty-five patients with esophageal cancer undergoing transthoracic esophagectomy between June 2017 and April 2018 participated in this study. One patient was excluded from the analysis due to conversion to a twostaged procedure intraoperatively. Intraoperative body fluid management and anesthesia consisting of inhaled general anesthesia and epidural anesthesia depended on the preference of the anesthesiologist. The overall goal of postoperative body fluid management was to maintain the mean blood pressure above $65 \mathrm{mmHg}$ mainly by administering crystalloids, and albumin solutions and/or circulating agonists when the mean blood pressure did not respond to sufficient crystalloid administration. Red blood cell (RBC) transfusions were considered when the patient's hemoglobin levels were lower than $8.0 \mathrm{~g} / \mathrm{dL}$ at the perioperative periods.

Pathological staging was based on the Union for International Cancer Control (UICC) TNM staging system 8th edition for esophageal cancer [ 16]. Physical status before surgery was classified according to the system of the American Society of Anesthesiologists [17]; ASA-PS. Nutritional status before surgery was also evaluated using Prognostic Nutritional Index (PNI) by Onodera et al. [18] because of the possibility of influencing the postoperative course [19]. Postoperative complications were assessed using the Clavien-Dindo classification (CD classification) [20]. We employed a prospective observational study design, at a single center, and obtained approval from the ethics committee of the Faculty of Medicine, The University of Tokyo.

\section{Recording body weights and cumulative fluid balances}

Body weights and the cumulative fluid balances of the patients were recorded as the reference indicators in this study. Body weights were recorded once daily from the 1 st to the 7th postoperative day (POD). Cumulative fluid balances (CFB) were calculated as the difference between the total fluid intakes and the outputs divided by the preoperative body weights. Insensible perspirations were not taken into account. The CFBs were calculated directly after the surgery and once daily at 12:00 from POD 1 to 7 .

\section{BIA measurements}

The BIA measurements were performed using a multifrequency bioelectrical impedance analysis system (MLT-550 N, SK Medical Electronics Co., Ltd., Tokyo, Japan). BIA uses a low alternating electric current that does not affect the subject unless embedded devices, such as cardiac pacemakers, are present. The alternating electric current with low frequency tends to flow only through the extracellular fluid compartment, while the 
high frequency current flows to both the extracellular and the internal cellular fluid compartments. The resistance values which reflect opposition to the current flow through intra- and extracellular fluids, and the reactance values which reflect the capacity produced by interfaces of tissues and cell membranes [6], are both measured using these alternating electric currents with multiple frequencies. From the obtained resistance and reactance values and the subject's height and other general information, the amounts of extracellular and internal cellular fluids contained in the subject's body are calculated.

The patients were instructed to remain in the supine position with their upper limbs not touching their trunk and with the thighs also kept apart. Two electrodes were attached to the back of the right wrist and another two on the right foot. The measurements were carried out using alternating currents up to 250 micro amps with multiple frequencies ranging from $2.5 \mathrm{kHz}$ to $350 \mathrm{kHz}$. The amounts of extracellular water (ECW), internal cellular water (ICW) and total body water (TBW) were calculated from the obtained resistance value, reactance value, sex and height information. The body weight was additionally required to calculate fat-free mass (FFM). The body weight one hour after surgery was estimated from the preoperative body weight and the cumulative fluid balance during surgery. When the body weight value was not available at other measurement timepoints, the weight just prior to the measurement was applied for the FFM calculations. These estimated body weight values were not used for analyzing postoperative body weight trends.

The baseline BIA was measured two days before surgery and one hour after surgery, followed by two daily measurements in the morning (around 10:00) and evening (around 18:00) from POD 1 to 7. It was not recorded when the patient was under management with a life support device other than a mechanical ventilator or a hemodialysis device, nor when the patient refused the measurement due to physical discomfort after surgery or for any other reason.

\section{Estimations of fluid retention timings}

The onset of initial fluid retention was defined as the first day of two consecutive days of weight loss after surgery, as conventionally estimated. Weight loss timing was compared with the peak change in each parameter obtained by BIA measurements in each patient.

\section{Definition of infectious complications}

Major postoperative adverse events were recorded after the surgery and we defined postoperative pneumonias, anastomotic leakages, and other surgical site infections of C-D grade 2 or higher as the infectious complications. Diagnostic criteria for pneumonias included pulmonary infiltrative shadows on the chest X-rays or Computerized tomographic scannings (CTs), purulent sputum, and increased white blood cell (WBC) counts or C-related protein (CRP) levels. Anastomotic leakages and other surgical site infections were confirmed by CT or contrast agent test when both of the purulent effluent from drainage tubes and the elevation in WBC counts or CRP levels were observed. We divided the patients into two groups based on the occurrence of these infectious adverse events in an effort to identify differences between the groups in postoperative BIA parameter trend changes. The changing trends of the body weights and the CFBs were also compared between these groups.

\section{Statistical analysis}

Statistical analyses were all performed with JMP Pro 13.2 software (SAS Institute Inc. Cary, NC, USA). Continuous variables were compared between groups by the Man-Whitney $U$ test. In the logistic analyses, Fisher's exact tests were used for categorical variables. Cut-off values for the receiver operating characteristics (ROC) curves were determined by Youden index. For the multivariate analyses, linear regression analyses using leastsquares methods and multivariate logistic regressions with forced entry methods were carried out. $P$ values less than 0.05 were considered to indicate a statistically significant difference.

\section{Results}

\section{Changing trends in TBW, ECW and ICW}

In total, 372 BIA measurements were performed in the 24 patients. Figure 1a shows the mean amounts of change in TBW, ECW and ICW from the preoperative levels (TBW-C, ECW-C and ICW-C) in our 24 patients. The mean ECW-C peaked on POD 1 to 2, whereas the mean ICW-C remained at approximately the preoperative level throughout the measurement period. Thus, the mean TBW-C, calculated as the sum of ECW-C and ICW-C, also peaked on POD1 to 2.

\section{Changing trends in TBW, ECW and ICW relative to FFM}

Figure $1 \mathrm{~b}$ shows the changes in the proportions of each body water compartment to FFM: ECW/FFM-C, ICW/ FFM-C and TBW/FFM-C. The ECW/TBW (ECW/ TBW-C) change is also shown. The mean ECW/FFM-C and TBW/FFM-C peaked, while the mean ICW/FFM-C reached the lowest value on POD1 to 2. TBW/FFM-C was lower than ECW/FFM-C and the mean ECW/TBW$\mathrm{C}$ was equivalent to the mean ECW/FFM-C throughout the measurement period. 


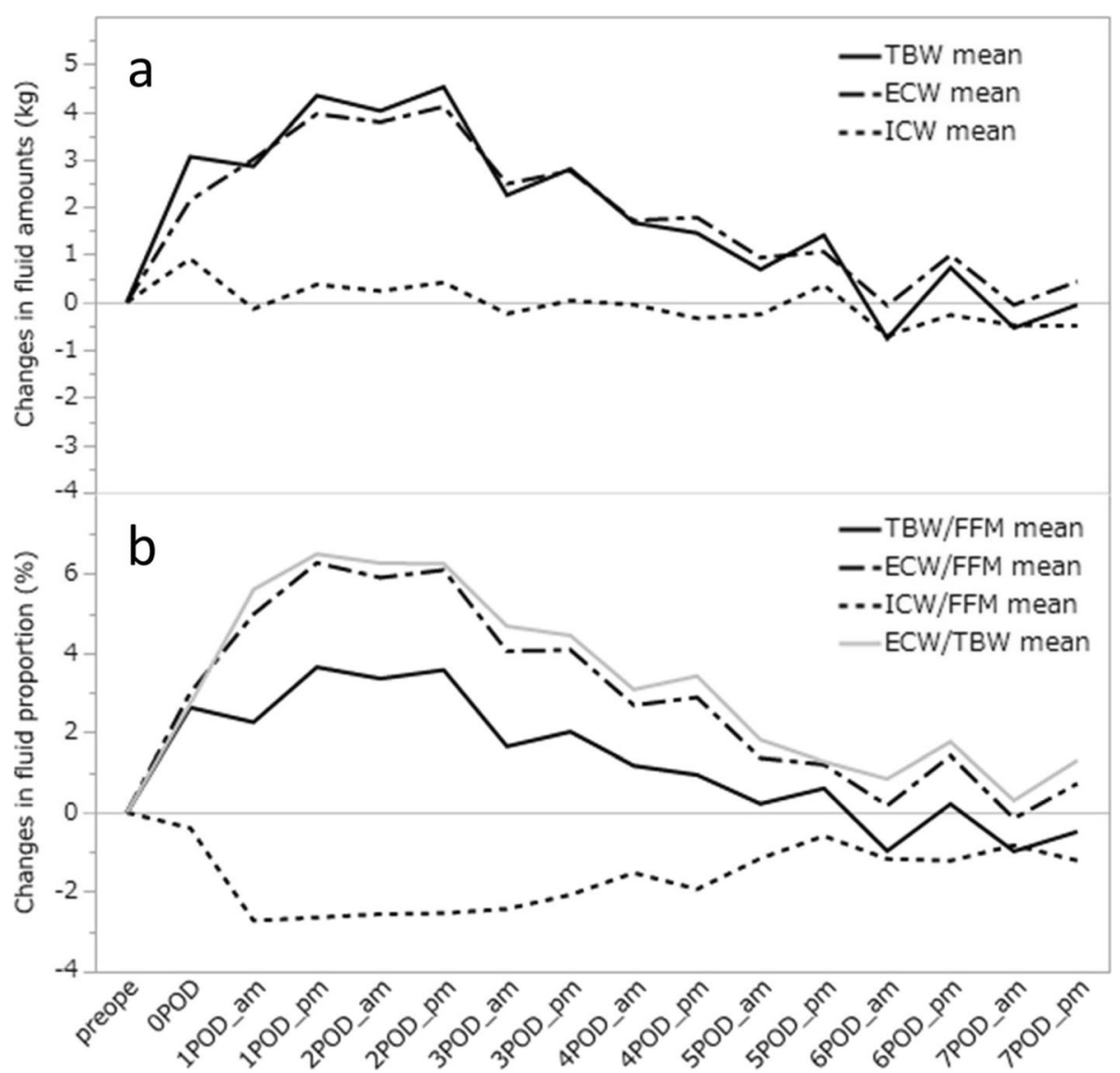

Fig. 1 Changes in body fluid amounts (a) and changes in fluid proportions in patients after transthoracic esophagectomy (b). Increments from the preoperative state are shown for one week after surgery. TBW, total body water; ECW, extracellular water; ICW, internal cellular water; FFM, fat free mass

Peaks of BIA parameters and their relationships with body fluid retention

The times of fluid retention were not defined in three cases since the body weight trend patterns did not meet the criteria. In most of the remaining 21 patients, the times of the maximum values in ECW, ECW/FFM and ECW/TBW appeared on the same day, or 1-2 days prior to the day that fluid retention manifested, while appearing a few days later in the others (Additional file 1: Figure S1). Correspondingly, the ICW/FFM minimum peaks appeared within two days before and after the day that fluid retention became apparent.

\section{Postoperative adverse events and their relationships with clinical factors}

Table 1 shows the numbers of patients who experienced major postoperative adverse events and their incidence rates. Incidence rate of the pneumonias, leakages and other surgical site infections of C-D grade 2 or more were $37.5,12.5$ and $12.5 \%$, respectively. These infectious complications developed in 12 patients including 4 who experienced multiple adverse events, and they were observed on POD3 or later except in three patients with pneumonia which developed on POD2. Two cases of acute respiratory distress syndrome (ARDS) were all accompanying other infectious complications.

The patients were then divided into an infection group $(n=12)$ and a non-infection group $(n=12)$ in accordance with their complication occurrence status (Table 2). None of the factors listed differed significantly between the two groups.

\section{Relationship between infection and change in body weight, cumulative fluid balance}

The trends of body weight changes from the preoperative levels are shown in Fig. 2a. Body weights peaked on POD1 in the non-infection group whereas the peak was on POD3 in the infection group, and the increment in the infection group was also higher than that of the noninfection group. The median value (25th percentile, 75 th percentile) of Body weight changes was significantly higher in the infection group than in the non-infection 
Table 1 Incidence rates of postoperative adverse events observed in patients after open esophagectomy

\begin{tabular}{|c|c|c|c|}
\hline Total & & $n=24$ & (\%) \\
\hline \multirow[t]{3}{*}{ Pneumonia } & Grade2 & 6 & $25.0 \%$ \\
\hline & $>=$ Grade3a & 3 & $12.5 \%$ \\
\hline & Any & 9 & $37.5 \%$ \\
\hline ARDS & Any & 2 & $8.3 \%$ \\
\hline \multirow[t]{3}{*}{ Leakage } & Grade2 & 1 & $4.2 \%$ \\
\hline & $>=$ Grade $3 \mathrm{a}$ & 2 & $8.3 \%$ \\
\hline & Any & 3 & $12.5 \%$ \\
\hline \multirow[t]{4}{*}{ SSI } & Wound & 1 & $4.2 \%$ \\
\hline & Mediastinitis & 1 & $4.2 \%$ \\
\hline & Empyema & 1 & $4.2 \%$ \\
\hline & Any & 3 & $12.5 \%$ \\
\hline \multirow[t]{3}{*}{ Chylothorax } & Grade2 & 1 & $4.2 \%$ \\
\hline & $>=$ Grade3a & 1 & $4.2 \%$ \\
\hline & Any & 2 & $8.3 \%$ \\
\hline Pleural fluid & $>=$ Grade $3 \mathrm{a}$ & 6 & $25.0 \%$ \\
\hline Pneumothorax & $>=$ Grade3a & 1 & $4.2 \%$ \\
\hline Laryngeal N paralysis & $>=$ Grade $3 \mathrm{a}$ & 1 & $4.2 \%$ \\
\hline Delayed Emptying & $>=$ Grade 2 & 3 & $12.5 \%$ \\
\hline Af & Any & 9 & $37.5 \%$ \\
\hline
\end{tabular}

Infection-related events are shaded in the table. Severities of the adverse events are graded according to the Clavien-Dindo classification. Some cases had experienced overlapping episodes. ARDS Acute Respiratory Distress Syndrome, $S S /$ surgical site infection, Laryngeal $N$ laryngeal nerve, $A f$ Atrial fibrillation

group on POD2 $[+3.19 \mathrm{~kg}(+2.81,+4.26) \mathrm{vs}+1.75 \mathrm{~kg}$ $(0.75,3.48), p=0.0376]$ and POD $4[+2.43 \mathrm{~kg}(+1.08,+$ $5.70) \mathrm{vs}+0.33 \mathrm{~kg}(-1.02,2.39), p=0.0282]$ or later.

CFBs were also higher in the infection group throughout the week after the surgery (Fig. 2b), and the values were significantly higher in the infection group than the non- infection group on POD3 $[+113.9 \mathrm{~mL} / \mathrm{kg}(+72.4$, $+157.2) \mathrm{vs}+70.9 \mathrm{~mL} / \mathrm{kg}(+51.9,+110.2), p=0.0327]$, on POD4 $[+117.6 \mathrm{~mL} / \mathrm{kg}(+87.4,+169.7) \mathrm{vs}+77.2 \mathrm{~mL} / \mathrm{kg}$ $(+39.1,+102.0), p=0.0284]$, and on POD5 $[+124.4 \mathrm{~mL} /$ $\mathrm{kg}(+88.5,+158.8) \mathrm{vs}+89.9 \mathrm{~mL} / \mathrm{kg}(+37.3,+114.4), p=$ $0.0449]$.

\section{Relationship between infection and change in ECW amount}

The measured amount of ECW peaked in the evening of POD1 in the non-infection group and in the evening of POD2 in the infection group (Fig. 3a). In addition, the increment in the peak from the preoperative level was greater in the infection group (Fig. 3b). The median value (25th percentile, 75 th percentile) of ECW-C was significantly higher in the infection group than in the non-infection group in the morning of POD2 $[+3.90 \mathrm{~kg}$ $(+3.40,+7.80)$ vs $+2.40 \mathrm{~kg}(+1.75,+3.45), p=0.0081]$, in the evening of POD2 $[+5.15 \mathrm{~kg}(+3.98,+8.35) \mathrm{vs}+$ $2.15 \mathrm{~kg}(+0.83,+3.95), p=0.0042]$, and in the morning of POD3 $[+3.20 \mathrm{~kg}(+1.70,+5.20)$ vs $+1.05 \mathrm{~kg}(-0.38$, $+2.60), p=0.0209]$.

Considering the possible predictors listed on Table 2 except for the pathological status of the resected tumor, linear regression analyses for the ECW-C values on POD2 revealed that the patients who received intraoperative $\mathrm{RBC}$ transfusion and the patients without any neoadjuvant therapy tended to have higher ECW-C values in the morning of POD2 ( $p=$ 0.0220 and $p=0.0287$, respectively), and the elderly patients also had higher ECW-C values in the evening of POD2 $(p=0.0337)$.

\section{Relationship between infection and the ICW/FFM value} The median value (25th percentile, 75 th percentile) of ICW/FFM was significantly lower in the infection group throughout the BIA measurement period, even before surgery, but most remarkably in the morning of POD2 [35.5\% $(34.2,36.2)$ vs $38.9 \%$ (38.2, 41.8), $p=$ 0.0004] (Fig. 4a). In contrast, the ICW/FFM-C values, for the most part, did not differ between the groups during the measurement period except in the evening of POD2 (Fig. 4b).

Applying linear regression analyses for the ICW/FFM values on POD2 as the same way as for the ECW-C values, we found that female patients had higher ICW/ FFM values than male patients only in the evening of POD2 $(p=0.0489)$. No other factors were relevant to the ICW/FFM values observed at any time points.

\section{Relationship between infection and the ECW/TBW value} The median value (25th percentile, 75 th percentile) of ECW/TBW was higher in the infection group than in the non-infection group throughout the measurement period, and statistical significance was initially recognized in the morning of POD2 $[50.2 \%(47.8,55.3)$ vs $46.2 \%(41.9,48.5), p=0.0106$ ] (Fig. 5a). It peaked in the evening of POD1 in the non-infection group, while the highest value was much greater in the infection group and appeared later on POD2 when ECW/TBW values already showed a decreasing trend in the non-infection group. The difference in ECW/TBW-C between the two groups was also significant in the evening of POD2 (Fig. 5b), but it was not as remarkable as the difference in the measured ECW/TBW values.

Linear regression analyses revealed that none of the factors listed on Table 2 affected the ECW/TBW values on POD2 at any time points. 
Table 2 Comparison of patient characteristics with or without the infectious postoperative complications after transthoracic esophagectomy

\begin{tabular}{|c|c|c|c|c|c|}
\hline & & \multirow[t]{2}{*}{ All $(n=24)$} & \multicolumn{2}{|c|}{ Infectious $A E,>=$ Grade2 } & \multirow{2}{*}{$\begin{array}{l}P \\
\text { value }\end{array}$} \\
\hline & & & yes $(n=12)$ & no $(n=12)$ & \\
\hline Sex & Male / Female & $17 / 7$ & $10 / 2$ & $7 / 5$ & 0.3707 \\
\hline Age & Mean \pm SD & $68.1 \pm 7.7$ & $70.5 \pm 8.8$ & $65.8 \pm 6.4$ & 0.1698 \\
\hline Height (cm) & Mean \pm SD & $161.9 \pm 9.1$ & $163.0 \pm 8.6$ & $160.8 \pm 10.3$ & 0.7439 \\
\hline Preoperative BW (kg) & Mean \pm SD & $56.5 \pm 12.5$ & $55.1 \pm 13.6$ & $58.0 \pm 11.8$ & 0.4010 \\
\hline BMI $\left(\mathrm{kg} / \mathrm{m}^{2}\right)$ & Mean \pm SD & $21.3 \pm 3.4$ & $20.6 \pm 4.0$ & $22.0 \pm 2.7$ & 0.4862 \\
\hline \multirow[t]{2}{*}{ Preoperative therapy } & none & 5 & 3 & 2 & \multirow[t]{2}{*}{1.0000} \\
\hline & Chemo/CRT & $14 / 5$ & $6 / 3$ & $8 / 2$ & \\
\hline ASA-PS & $<=1 />=2$ & $7 / 17$ & $4 / 8$ & $3 / 9$ & 1.0000 \\
\hline PNI (Onodera et al.) & Mean \pm SD & $44.6 \pm 5.7$ & $44.5 \pm 6.0$ & $44.8 \pm 5.8$ & 0.9323 \\
\hline Reconstruction route & ITH / PMD / RST & $10 / 10 / 4$ & $6 / 5 / 1$ & $4 / 5 / 3$ & 0.6634 \\
\hline Lymph node dissection & $<=2 / 3$ fields & $12 / 12$ & $7 / 5$ & $5 / 7$ & 0.6843 \\
\hline Operative time (min) & Mean \pm SD & $403.3 \pm 54.7$ & $394.8 \pm 46.6$ & $411.8 \pm 64.8$ & 0.3259 \\
\hline Intraoperative Blood Loss (mL) & Mean \pm SD & $513.3 \pm 271.4$ & $520.4 \pm 285.9$ & $506.3 \pm 268.6$ & 0.9081 \\
\hline \multirow[t]{2}{*}{$\mathrm{RBC}$ transfusion intraoperative early postope, $\sim 2 \mathrm{POD}$} & No/Yes & $23 / 1$ & $11 / 1$ & $12 / 0$ & 1.0000 \\
\hline & No/Yes & $17 / 7$ & $8 / 4$ & $9 / 3$ & 1.0000 \\
\hline Steroid use ( 7POD) & No/Yes & $17 / 7$ & $7 / 5$ & $10 / 2$ & 0.3707 \\
\hline Sivelestat use ( 7POD) & No/Yes & $16 / 8$ & $6 / 6$ & $10 / 2$ & 0.1930 \\
\hline Resection & $\mathrm{R} 0,1 / \mathrm{R} 2$ & $20 / 4$ & $11 / 1$ & $9 / 3$ & 0.5901 \\
\hline \multirow[t]{2}{*}{ pT } & $0 / 1 \mathrm{a} / 1 \mathrm{~b} / 2$ & $1 / 2 / 2 / 4$ & $1 / 1 / 0 / 1$ & $0 / 1 / 2 / 3$ & \multirow[t]{2}{*}{0.4003} \\
\hline & $3 / 4$ & $12 / 3$ & $8 / 1$ & $4 / 2$ & \\
\hline \multirow[t]{2}{*}{$\mathrm{pN}$} & 0 & 4 & 2 & 2 & \multirow[t]{2}{*}{1.0000} \\
\hline & $1 / 2 / 3$ & $14 / 2 / 4$ & $6 / 2 / 2$ & $8 / 0 / 2$ & \\
\hline $\mathrm{pM}^{\mathrm{a}}$ & $0 / 1$ & $23 / 1$ & $12 / 0$ & $11 / 1$ & 1.0000 \\
\hline
\end{tabular}

No patient, surgical or pathological factors, related to the complication incidence, were identified. $\mathrm{pM}^{\mathrm{a}}$ includes metastasis involving the supraclavicular lymphnodes. AE Adverse Events, BW body weight, BMI body mass index, PNI Prognostic Nutritional Index (preoperatively calculated), ASA-PS Physical state classification by the American Society of Anesthesiologists, ITH intrathoracic route, PMD post-mediastinal route, RST retrosternal route, RBC Red Blood Cell, postope postoperative, $p T / N / M$ pathological T/N/M

ROC analyses of BIA parameters, body weight changes, and $C F B s$ regarding postoperative infection

As ECW-C, ICW/FFM and ECW/TBW values were observed to be significantly different according to the presence of infectious complications on POD2, ROC curve analyses were employed to evaluate the performance of each value for diagnosing infectious adverse events. The cut-off values and the values of the area under these ROC curves (AUROC) in the morning and evening are shown in Table 3. As the references, ROC curve analyses were also employed for the body weight changes and the CFBs in the same manner using the data on POD2. The AUROC of ICW/FFM in the morning of POD2 was the highest, at 0.93 , with a cut-off value of $36.7 \%$. That in the evening of POD2 was also high, at 0.83 , with a cutoff value of $37.2 \%$. The remaining AUROCs of ECW-C and $\mathrm{ECW} / \mathrm{TBW}$ were equivalently high, employing the optimal cut-off value for each. All of these AUROC values were better than those calculated from the body weight changes and CFBs.

\section{Multivariate logistic regression analyses of BIA parameters on POD2}

Multivariate logistic analyses were also carried out to prove whether the measured BIA values on POD2 could be considered as the independent predictors of the morbidity. The measured BIA values on POD2 except for ICW/FFM in the morning were independent significant predictors for the infectious complications (all $p<0.01$ ), while the patient factors (sex, age, preoperative body mass index (BMI), neoadjuvant therapy, ASA-PS, and preoperative $\mathrm{PNI}$ ) and the surgical factors (lymph node dissection, reconstruction route, operative time, intraoperative blood loss, intra-operative $\mathrm{RBC}$ transfusion, and resection margin) were not statistically significant. 


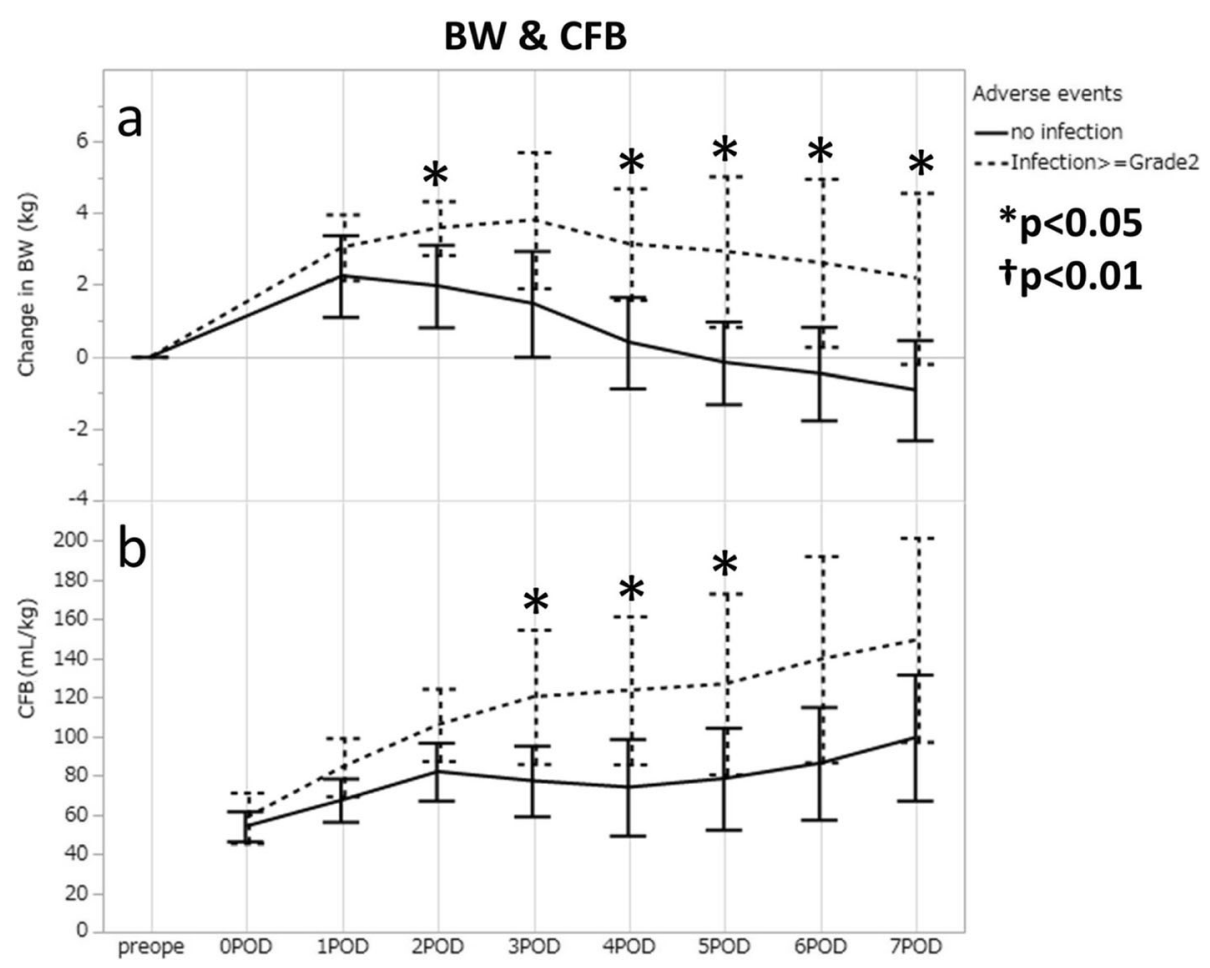

Fig. 2 Changes in body weight increments (a) and the cumulative fluid balances (b) after open esophagectomy in patients, with and without infectious complications. Insensible perspirations were not taken into account for the CFB calculations. The mean values and the $95 \%$ confidential intervals are presented to show the trends within the same groups. BW, body weight; CFB, cumulative fluid balance. ${ }^{*} p<0.05,+p=0.01$ by MannWhitney $U$ test

\section{Discussion}

We herein evaluated the changes in water distribution in patients undergoing transthoracic esophagectomy. The $\mathrm{ECW}$ value rose overall for 2 days, decreasing thereafter and reaching the baseline 6-7 days after esophagectomy, while the ICW value remained stable throughout the postoperative course. This observation is highly consistent with the phenomenon known as "third spacing", representing the fluid shift from the intravascular to the nonfunctional interstitial space. It implies a reduced blood volume and increased fluid in the interstitial area, i.e. tissue edema, and is essentially associated with reduced cardiac output and systemic hypoperfusion unless appropriate fluid replacement is given to maintain the patient's blood pressure. Therefore, fluid resuscitation appears to be required for the initial 2 days and fluid restriction to prevent fluid overload in the subsequent postoperative days. This is the first report, to our knowledge, demonstrating the trends in BIA parameters measured for one week after transthoracic esophagectomy. The ECW-related values (ECW/FFM and ECW/TBW) peaked at the conventionally estimated onset of the fluid retention, or even earlier, in some patients. The BIA measurements appear to be useful for predicting and confirming fluid retention.
Transthoracic esophagectomy procedures encompass a broad range of surgical fields including the neck, thoracic and abdominal cavities. Moreover, direct compressions of the patient's right lung or heart, and also onelung ventilation can damage the cardiopulmonary system, especially during the transthoracic procedure. Transthoracic esophagectomy is clearly one of the most invasive surgeries. It is associated with enlargement of the interstitial fluid compartment, thereby necessitating very careful fluid management.

ECW-C was more than $+4 \mathrm{~kg}$ on POD2 on average, and the amount as well as the onset of the peak differed according to the presence of infectious complications. It is noteworthy that the ECW and ECW/TBW, which had increased, were restored to their baseline values on POD5 in patients without infectious complications, while remaining above baseline values until POD7 in those with infections. ECW values were generally very high in critically ill patients, especially those with sepsis [21]. ECW excess was not diminished in non-survivors with sepsis, in contrast to ECW which was significantly decreased from the baseline in surviving sepsis patients [22]. These observations might support our results, at least in part, indicating sustained increases in ECW over the baseline to be associated with sustained systemic 


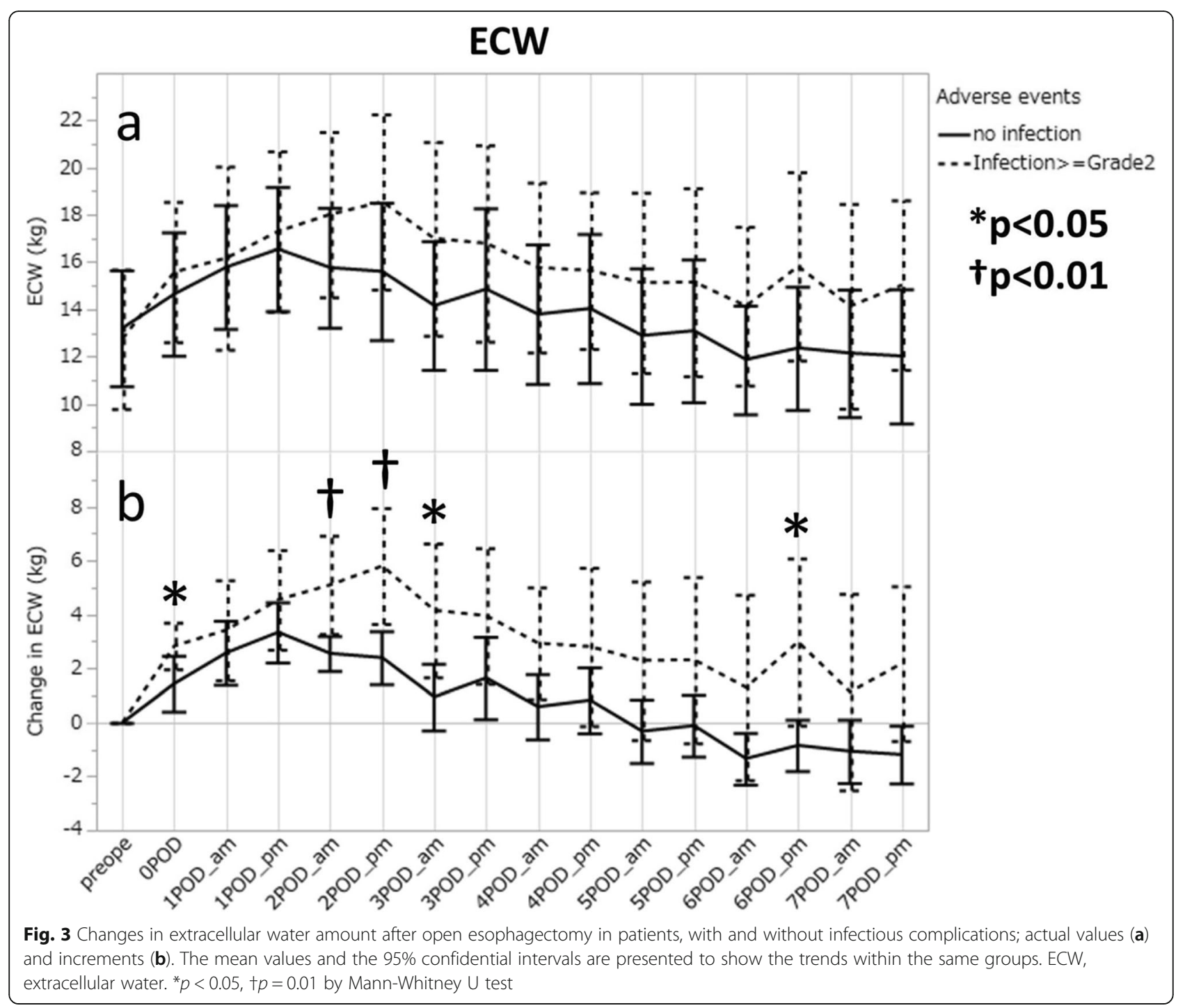

inflammation followed by the development of infectious complications.

The ECW-C and ECW/TBW values differed significantly, especially on POD2, regardless of whether or not infectious complications developed. This might be attributable to infectious adverse events being associated with an increase in the interstitial fluid compartment, potentially leading to an increased demand for infusion therapy to maintain hemodynamics before the complications become clinically evident. This implies that postoperative infections might be the mechanism underlying abnormal excessive ECW storage before these adverse events manifest clinically during the postoperative course.

Fluid management strategies reportedly affect the incidence of postoperative adverse events. Fluid restriction and early-goal-directed therapy reportedly produce more favorable post-surgical outcomes than administering higher volumes of fluid without hemodynamic goals [4, 5]. A stratified meta-analysis showed a lower incidence of pneumonia and shorter ICU stays with goal-directed fluid management than with less strict fluid management protocols [5]. Stroke-volume guided goal-directed fluid management was also reported to reduce the occurrence of pneumonia, mediastinal abscesses and gastric tube necrosis, as well as shortening the ICU stay for postesophagectomy patients [23]. We did not employ the "restricted" fluid management strategy and thus could not rule out the possibility of a causal relationship between excessive fluid infusion and infections in our prospective observational study, although we did not change the postoperative fluid management strategy for our patients. The CFB values between the infection and noninfection groups were significantly different only from POD 3 to 5, which appeared later than the significant difference in the ECW related parameters and also later 


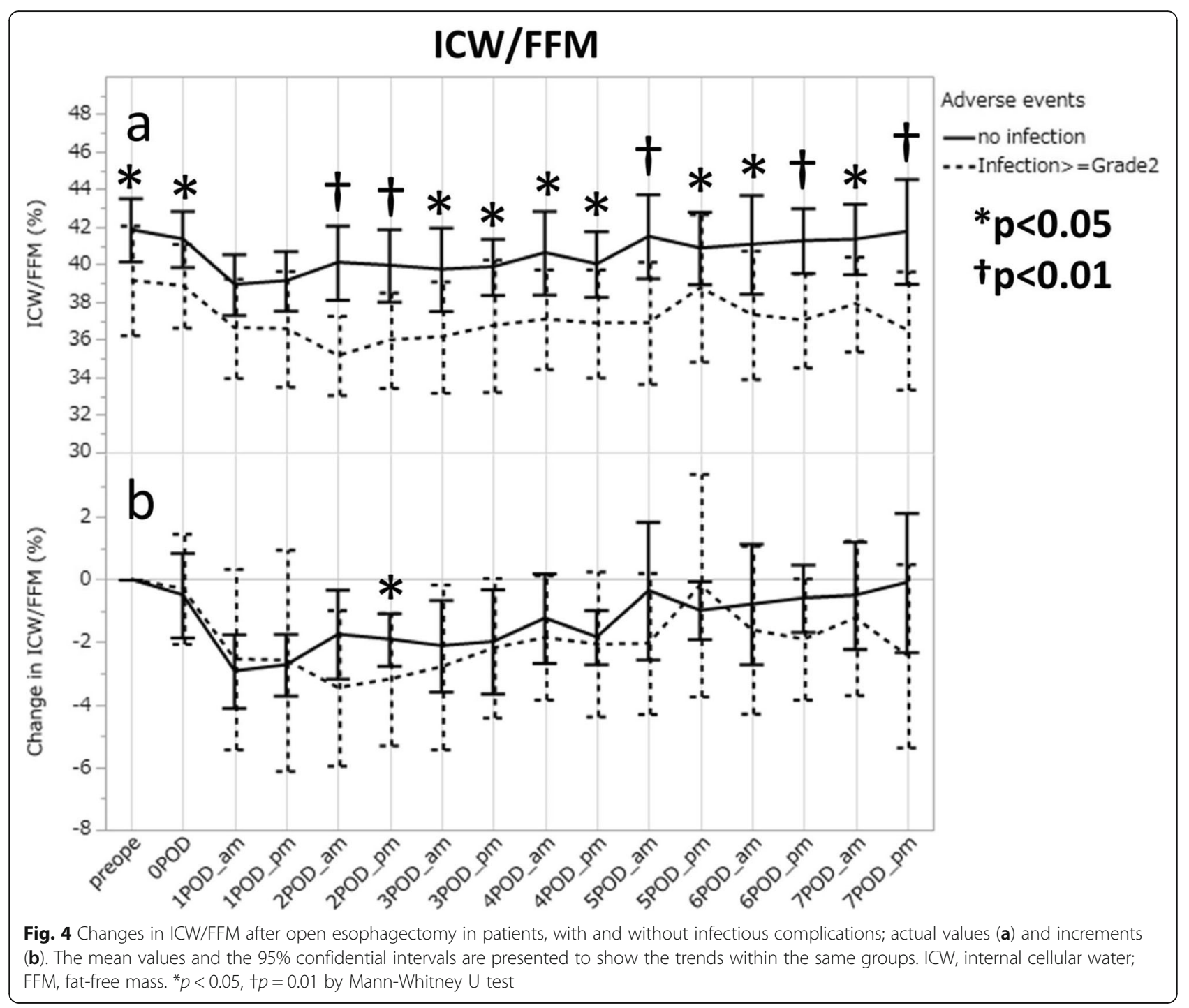

than the occurrences of any infectious complications. The trends in BIA measurement changes according to the fluid management strategy and type of intravenous fluid (crystalloid or colloid) warrant further study.

ICW/FFM was the most predictive parameter with its high AUROC of 0.93 in the morning of POD2 when the cut-off value was set at $36.7 \%$, although it was significantly lower in the infection group throughout almost the entire measurement period. One plausible explanation is that the excessive increase in the ECW compartment was significantly greater in the infection group. However, given that the ICW/FFM values were significantly lower in the infection group before and just after surgery, we can speculate that the baseline characteristics might have differed between the two groups. Studies have shown multi-frequency BIA to be useful for estimating muscle cross-sectional area [24] and strength as well. ICW amounts are known to correlate with the skeletal muscle mass, while ECW shows an inverse correlation. The ECW/ICW ratio in skeletal muscle is inversely correlated with gait speed independently of age, sex, body mass index and skeletal muscle mass [25], suggesting a positive relationship between low ICW amount and sarcopenia status. In addition, ICW shows an inverse correlation with frailty [26], such that a low ICW/ FFM value at baseline might indicate sarcopenia and/or frailty affecting patients in the infection group. Studies have suggested sarcopenia to be a predictor of postoperative respiratory adverse events after esophagectomy [ $27,28]$, observations which may support our aforementioned inferences.

Our results might reflect the mechanisms by which BIA parameters change, with or without infectious complications, after esophagectomy as shown in Additional file 2: Figure S2; secondary fluid excess in response to the infectious complications was confirmed by the significant 


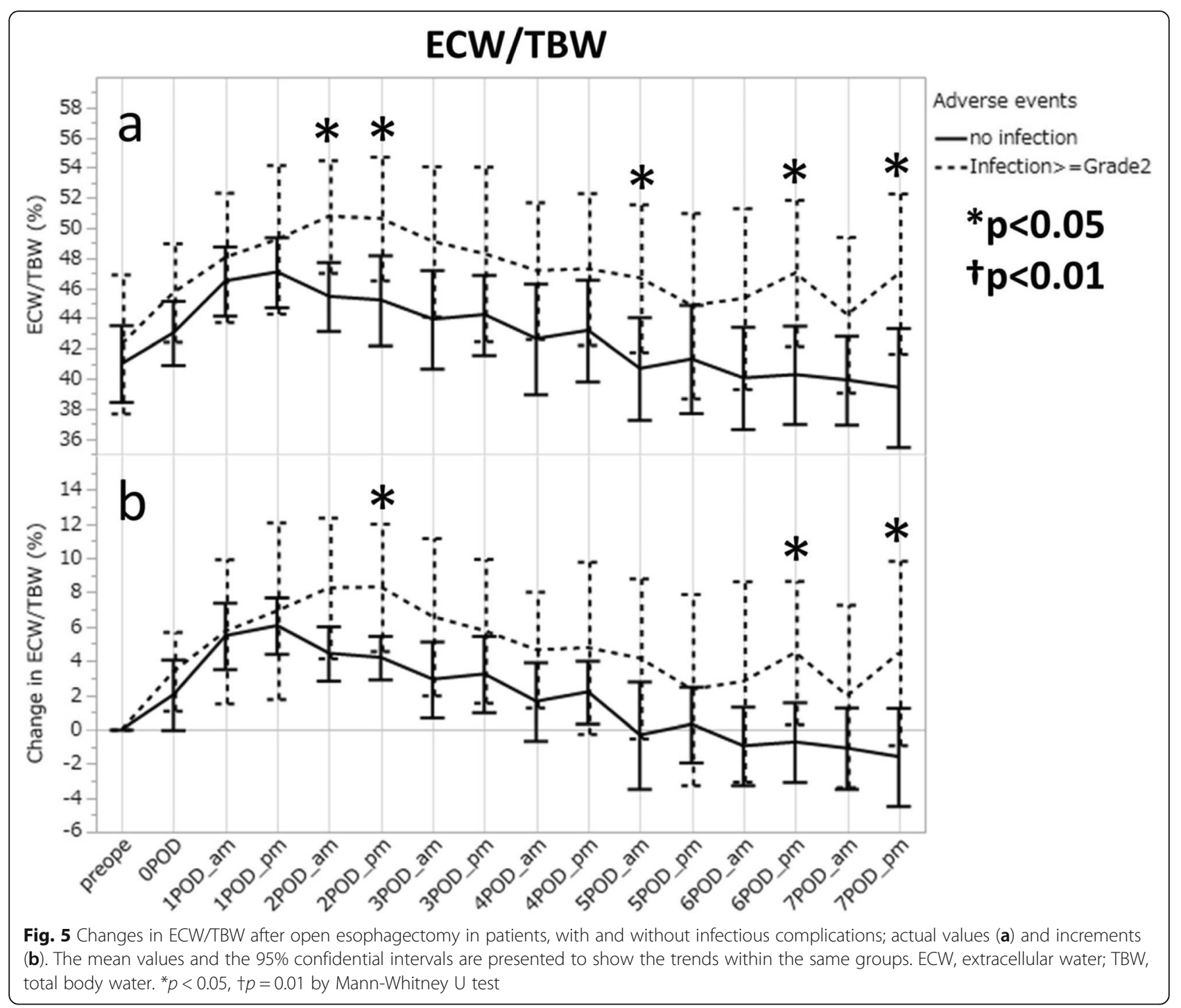

Table 3 ROC analysis in the prediction of infectious complications with each parameter on POD 2

\begin{tabular}{|c|c|c|c|c|c|}
\hline Parameter & Timing & AUROC & Cut-off value & Sensitivity & Specificity \\
\hline \multirow[t]{2}{*}{ ECW change } & 2POD am & 0.83 & $+3.0 \mathrm{~kg}$ & $90.7 \%$ & $66.7 \%$ \\
\hline & 2POD pm & 0.84 & $+3.9 \mathrm{~kg}^{\mathrm{a}}$ & $83.3 \%^{\mathrm{a}}$ & $75.0 \%^{\mathrm{a}}$ \\
\hline \multirow[t]{2}{*}{ ICW/FFM } & 2POD am & 0.93 & $36.7 \%$ & $90.9 \%$ & $100 \%$ \\
\hline & 2POD pm & 0.83 & $37.2 \%$ & $83.3 \%$ & $83.3 \%$ \\
\hline \multirow[t]{2}{*}{ ECW/TBW } & 2POD am & 0.81 & $47.8 \%$ & $81.8 \%$ & $75.0 \%$ \\
\hline & 2POD pm & 0.80 & $49.0 \%$ & $75.0 \%$ & $83.3 \%$ \\
\hline BW change & $2 \mathrm{POD}$ & 0.75 & $+2.5 \mathrm{~kg}$ & $91.7 \%$ & $66.7 \%$ \\
\hline CFB/ preop.BW & $2 \mathrm{POD}$ & 0.72 & $+101.1 \mathrm{~mL} / \mathrm{kg}$ & $75.0 \%$ & $66.7 \%$ \\
\hline
\end{tabular}

The cut-off values and the sensitivity and specificity values are shown with AUROC values

a Setting the cut-off value at $+4.2 \mathrm{~kg}$ also achieved a sensitivity of $75.0 \%$ and a specificity of $83.3 \%$. All cut off values were defined by Youden index. AUROC Area under the Receiver Operating Characteristics curve, ECW extracellular water, ICW internal cellular water, FFM fat free mass, TBW total body water, BW body weight, CFB cumulative fluid balance, preop preoperative 
increase in ECW, delayed peak and sustained increase in ECW in the presence of such complications. Furthermore, low preoperative ICW/FFM values before surgery exacerbated the increase in the ratio of ECW to TBW or FFM. It might be feasible to establish a better fluid management strategy based on the BIA parameters measured, serving as surveillance targets during the perioperative period. The multivariate analysis results in this study will also support the adequacy of this management strategy, although the BIA parameter values can be affected by the patient's age, sex, and the status of neoadjuvant therapy and intraoperative RBC transfusion.

This study has limitations. First, the number of patients analyzed in each group was small, and statistical differences in BIA parameters might not reflect the actual effects of infectious complications. Second, patients with minimally invasive esophagectomy were not included in this study, and we therefore cannot identify differences in BIA measurements between the open and minimally invasive approaches. Finally, the patients were all Japanese and the cut-off ECW/TBW and ICW/FFM values for predicting infectious events may vary according to the ethnicity of the population.

\section{Conclusion}

The peaks of ECW and ECW/TBW values differ according to the development of various infectious complications, and the ECW/TBW and ICW/FFM values on POD2 predict infectious adverse events after transthoracic esophagectomy. BIA measurements appear to be useful after transthoracic esophagectomy not only for assessing fluid retention and possibly allowing more meticulous fluid management, but also for predicting postoperative infectious adverse events based on the very high AUROC values obtained.

\section{Supplementary information}

Supplementary information accompanies this paper at https://doi.org/10. 1186/s12893-019-0652-z.

Additional file 1: Figure S1.Comparison of the times of the appearance of the peaks of each parameter with the postoperative fluid retention time estimated from the body weight change after surgery. The onset of fluid retention was defined as the first day of consecutive weight loss for two days after surgery. TBW, total body water; ECF, extracellular water; ICW, internal cellular water; FFM, fat-free mass.

Additional file 2: Figure S2. Summary of the body water distribution in patients after transthoracic esophagectomy. The amount of ECW increases after surgery and peaks at approximately Day1-18:00 without infection, but when an infection is present the peak is seen later, around Day2-18:00, and the ECW volume also becomes even higher. Patients with low preoperative ICW/FFM might be more dramatically affected in terms of their ICW/FFM and ECW/TBW percentage by this ECW volume change, which may result in higher sensitivity of these parameters as predictors of infectious complications after esophagectomy. ECW, extracellular water; ICW, internal cellular water; FFM, fat free mass; TBW, total body water.

\section{Abbreviations}

Af: Atrial fibrillation; ARDS: Acute respiratory distress syndrome; ASA-

PS: Physical state classification by the American Society of Anesthesiologists; AUROC: Area under receiver operating characteristic curve; BIA: Bioelectrcial impedance analysis; BMI: Body mass index; BW: Body weight; C-D classification: Clavien-Dindo classification; CFB: Cumulative fluid balance; CRP: C-related protein; CT: Computerized tomographic scanning; ECW: Extracellular water; ECW/FFM-C: Change in ECW/FFM; ECW/TBWC: Change in ECW/TBW; ECW-C: Change in ECW; FFM: Fat-free mass; ICW: Internal cellular water; ICW/FFM-C: Change in ICW/FFM; ICW-C: Change in ICW; Laryngeal N: Laryngeal nerve; PNI: Prognostic nutritional index; POD: Postoperative day; pT/N/M: Pathological T/N/M; RBC: Red blood cell; ROC: Receiver operating characteristic; SSI: Surgical site infection; TBW: Total body water; TBW/FFM-C: Change in TBW/FFM; TBW-C: Change in TBW; UICC: The Union for International Cancer Control; WBC: White blood cell

\section{Acknowledgements}

Not applicable.

\section{Authors' contributions}

SO and YS made substantial contributions to conception and design of the study, and $\mathrm{HY}, \mathrm{KY}$, and SA also gave critically important advices for the research design. SO, RI, KK, and AT measured the BIA data and interpreted them. SO analyzed the BIA data regarding the infectious postoperative complications after esophagectomy, and was a major contributor in writing the manuscript. HY was also involved in drafting the manuscript and revising it critically for important intellectual content. All authors read and approved the final manuscript.

\section{Funding}

This study was supported by Management Expenses Grants and Donations to the University of Tokyo. The instruments used for the BIA measurements were purchased with this fund, and the statistical software license was also provided. All other costs for the design of the study and collection, analysis, and interpretation of data and in writing the manuscript were also helped by the funding.

\section{Availability of data and materials}

The datasets used and/or analyzed during the current study are available from the corresponding author on reasonable request.

\section{Ethics approval and consent to participate}

This study was approved from the ethics committee of the Faculty of Medicine, The University of Tokyo (reference number: 11483, approved on April 10, 2017). All patients gave written informed consents to participate in the study by submitting institutional consent forms.

Consent for publication

Not Applicable.

\section{Competing interests}

The authors declare that they have no competing interests.

\section{Author details}

${ }^{1}$ Department of Gastrointestinal Surgery, The University of Tokyo, 7-3-1, Hongo, Bunkyo-ku, Tokyo 113-8655, Japan. ²Department of Bariatric \& Metabolic Care, Graduate School of Medicine, The University of Tokyo, 7-3-1, Hongo, Bunkyo-ku, Tokyo 113-8655, Japan.

Received: 13 November 2018 Accepted: 26 November 2019 Published online: 02 December 2019

\section{References}

1. Jacob M, Chappell D, Rehm M. The 'third space' - fact or fiction? Best Pract Res Clin Anaesthesiol. 2009;23(2):145-57.

2. Rhodes A, Evans LE, Alhazzani W, Levy MM, Antonelli M, Ferrer R, et al. Surviving Sepsis campaign: international guidelines for Management of Sepsis and Septic Shock: 2016. Crit Care Med. 2017;45(3):486-552.

3. Takeuchi H, Miyata H, Gotoh M, Kitagawa Y, Baba H, Kimura W, et al. A risk model for esophagectomy using data of 5354 patients included in a Japanese nationwide web-based database. Ann Surg. 2014;260(2):259-66. 
4. Gao T, Li N, Zhang JJ, Xi FC, Chen QY, Zhu WM, et al. Restricted intravenous fluid regimen reduces the rate of postoperative complications and alters immunological activity of elderly patients operated for abdominal cancer: a randomized prospective clinical trail. World J Surg. 2012;36(5):993-1002.

5. Corcoran T, Rhodes JE, Clarke S, Myles PS, Ho KM. Perioperative fluid management strategies in major surgery: a stratified meta-analysis. Anesth Analg. 2012;114(3):640-51.

6. Kyle UG, Bosaeus I, De Lorenzo AD, Deurenberg P, Elia M, Gómez JM, et al. Bioelectrical impedance analysis--part l: review of principles and methods. Clin Nutr. 2004:23(5):1226-43.

7. Kyle UG, Bosaeus I, De Lorenzo AD, Deurenberg P, Elia M, Manuel Gómez J, et al. Bioelectrical impedance analysis-part II: utilization in clinical practice. Clin Nutr. 2004;23(6):1430-53.

8. Malbrain ML, Huygh J, Dabrowski W, De Waele JJ, Staelens A, Wauters J. The use of bio-electrical impedance analysis (BIA) to guide fluid management, resuscitation and deresuscitation in critically ill patients: a bench-to-bedside review. Anaesthesiol Intensive Ther. 2014:46(5):381-91.

9. Basso F, Berdin G, Virzi GM, Mason G, Piccinni P, Day S, et al. Fluid management in the intensive care unit: bioelectrical impedance vector analysis as a tool to assess hydration status and optimal fluid balance in critically ill patients. Blood Purif. 2013;36(3-4):192-9.

10. Gonzalez J, Morrissey T, Byrne T, Rizzo R, Wilmore D. Bioelectric impedance detects fluid retention in patients undergoing cardiopulmonary bypass. J Thorac Cardiovasc Surg. 1995;110(1):111-8.

11. Itobi E, Stroud M, Elia M. Impact of oedema on recovery after major abdominal surgery and potential value of multifrequency bioimpedance measurements. Br J Surg. 2006;93(3):354-61.

12. Shim HJ, Jang JY, Lee SH, Lee JG. The effect of positive balance on the outcomes of critically ill noncardiac postsurgical patients: a retrospective cohort study. J Crit Care. 2014;29(1):43-8.

13. Chong JU, Nam S, Kim HJ, Lee R, Choi Y, Lee JG, et al. Exploration of fluid dynamics in perioperative patients using bioimpedance analysis. J Gastrointest Surg. 2016;20(5):1020-7.

14. Tatara T, Tsuzaki K. Measurements of extracellular water volume by bioelectrical impedance analysis during perioperative period of esophageal resection. Masui. 1999:48(11):1194-201.

15. Hanaki N, Ishikawa M, Nishioka M, Kashiwagi Y, Miki H, Miyake H, et al. Bioelectrical impedance analysis to assess changes in body water compartments after digestive surgery. Hepatogastroenterology. 2006;53(71): 723-9.

16. Rice TW, Patil DT, Blackstone EH. 8th edition AJCC/UICC staging of cancers of the esophagus and esophagogastric junction: application to clinical practice. Ann Cardiothorac Surg. 2017;6(2):119-30.

17. Fitz-Henry J. The ASA classification and peri-operative risk. Ann R Coll Surg Engl. 2011;93(3):185-7.

18. Onodera T, Goseki N, Kosaki G. Prognostic nutritional index in gastrointestinal surgery of malnourished cancer patients. Nihon Geka Gakkai Zasshi. 1984;85(9):1001-5.

19. Filip B, Scarpa M, Cavallin F, Cagol M, Alfieri R, Saadeh L, et al. Postoperative outcome after oesophagectomy for cancer: nutritional status is the missing ring in the current prognostic scores. Eur J Surg Oncol. 2015;41(6):787-94.

20. Dindo D, Demartines N, Clavien PA. Classification of surgical complications: a new proposal with evaluation in a cohort of 6336 patients and results of a survey. Ann Surg. 2004;240(2):205-13.

21. Plank LD, Hill GL. Similarity of changes in body composition in intensive care patients following severe sepsis or major blunt injury. Ann N Y Acad Sci. 2000:904:592-602.

22. Dabrowski W, Kotlinska-Hasiec E, Schneditz D, et al. Continuous venovenous hemofiltration to adjust fluid volume excess in septic shock patients reduces intra-abdominal pressure. Clin Nephrol. 2014;82(1):41-50.

23. Veelo DP, van Berge Henegouwen MI, Ouwehand KS, Geerts BF, Anderegg MC, van Dieren S, et al. Effect of goal-directed therapy on outcome after esophageal surgery: a quality improvement study. PLoS One. 2017;12(3): e0172806.

24. Yamada Y, Ikenaga M, Takeda N, Morimura K, Miyoshi N, Kiyonaga A, et al. Estimation of thigh muscle cross-sectional area by single- and multifrequency segmental bioelectrical impedance analysis in the elderly. J Appl Physiol (1985). 2014;116(2):176-82.

25. Yamada Y, Yoshida T, Yokoyama K, Watanabe Y, Miyake M, Yamagata E, et al. The extracellular to intracellular water ratio in upper legs is negatively associated with skeletal muscle strength and gait speed in older people. J Gerontol A Biol Sci Med Sci. 2017;72(3):293-8.

26. Johansen KL, Dalrymple LS, Delgado C, Kaysen GA, Kornak J, Grimes B, et al. Association between body composition and frailty among prevalent hemodialysis patients: a US renal data system special study. J Am Soc Nephrol. 2014;25(2):381-9.

27. Boshier PR, Heneghan R, Markar SR, Baracos VE, Low DE. Assessment of body composition and sarcopenia in patients with esophageal cancer: a systematic review and meta-analysis. Dis Esophagus. 2018;31:1-11.

28. Ida S, Watanabe M, Yoshida N, Baba Y, Umezaki N, Harada K, et al. Sarcopenia is a predictor of postoperative respiratory complications in patients with esophageal Cancer. Ann Surg Oncol. 2015;22(13):4432-7.

\section{Publisher's Note}

Springer Nature remains neutral with regard to jurisdictional claims in published maps and institutional affiliations.
Ready to submit your research? Choose BMC and benefit from:

- fast, convenient online submission

- thorough peer review by experienced researchers in your field

- rapid publication on acceptance

- support for research data, including large and complex data types

- gold Open Access which fosters wider collaboration and increased citations

- maximum visibility for your research: over $100 \mathrm{M}$ website views per year

At BMC, research is always in progress.

Learn more biomedcentral.com/submissions 